\title{
Whole grain consumption is inversely associated with BMI Z-score in rural school-aged children
}

\author{
Silvina F Choumenkovitch ${ }^{1, *}$, Nicola M McKeown ${ }^{2}$, Alison Tovar ${ }^{1}$, Raymond R Hyatt ${ }^{3}$, \\ Vivica I Kraak ${ }^{4}+$, Alia V Hastings ${ }^{1}$, Julia Bloom Herzog ${ }^{1}$ and Christina D Economos ${ }^{1}$ \\ 'John Hancock Research Center on Physical Activity, Nutrition, and Obesity Prevention, Friedman School \\ of Nutrition Science and Policy, Tufts University, 150 Harrison Avenue, Boston, MA 02111 , USA: \\ ${ }^{2}$ Jean Mayer-USDA HNRCA at Tufts University, Boston, MA, USA: ${ }^{3}$ School of Medicine, Tufts University, \\ Boston, MA, USA: ${ }^{4}$ US Programs, Save the Children, Washington, DC, USA
}

Submitted 27 September 2011: Final revision received 18 April 2012: Accepted 17 June 2012: First published online 16 August 2012

\begin{abstract}
Objective: To examine the relationship between intake of whole grains and BMI $Z$-score in rural children.

Design: General linear models and logistic regression were used to examine the cross-sectional associations between whole grain intake and BMI Z-score, prevalence and odds ratios of overweight and obesity. Dietary intake was assessed using the Block Food Screener for ages 2-17 years. Children were classified into three categories according to servings of whole grain intake: $<1.0$ serving/d, $1 \cdot 0-1.5$ servings/d and $>1.5$ servings $/$ d.

Setting: The CHANGE (Creating Healthy, Active and Nurturing Growing-up Environments) study, an obesity prevention intervention in elementary schools in eight rural US communities in California, Mississippi, Kentucky and South Carolina. Subjects: Seven hundred and ninety-two children attending 3rd-6th grade.

Results: After adjusting for age, sex, race/ethnicity, physical activity and state of residence, whole grain intake was inversely associated with BMI Z-score (0.90 v. $0 \cdot 61$ in the lowest $v$. the highest whole grain intake category; $P$ trend $=0 \cdot 01$ ). Children who consumed $>1.5$ servings of whole grains/d had a $40 \%$ lower risk of being obese $(\mathrm{OR}=0 \cdot 60 ; 95 \% \mathrm{CI} 0 \cdot 38,0 \cdot 95, P=0 \cdot 02)$ compared with children who consumed $<1 \cdot 0$ serving/d. Further adjustment for potential dietary predictors of body weight (fruit, vegetable and dairy intakes) did not change the observed associations.

Conclusions: Increasing the intake of whole grains as part of an overall healthy lifestyle may be beneficial for children to achieve and maintain a healthy weight.
\end{abstract}

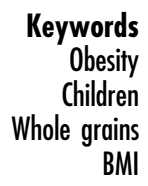

The prevalence of overweight and obesity has increased dramatically in the USA over the past three decades ${ }^{(1)}$. Currently, one-third of children aged 2-19 years are classified as overweight or obese $\mathrm{e}^{(2)}$. However, disadvantaged children living in rural areas are disproportionately at increased risk for overweight and obesity with prevalence rates reaching nearly $50 \%{ }^{(3-5)}$. Obesity in children is associated with physical and psychological health problems and obese children are more likely to be obese during adulthood compared with their normal-weight peers $^{(6)}$. In adults, obesity has been linked to an increased risk of type 2 diabetes, CVD, certain cancers, hypertension, dyslipidaemia, sleep apnoea, musculoskeletal disorders and psychosocial problems, among others ${ }^{(7-10)}$.

$\dagger$ Present address: Deakin Population Health Strategic Research Centre, School of Health and Social Development, Deakin University, 221 Burwood Highway, Burwood, Victoria 3125, Australia.
The soaring prevalence of overweight and obesity in addition to the health implications that obesity presents make identification of preventive lifestyle factors a priority. Significant inverse associations have been reported between the intake of wholegrain foods and body weight, weight gain and indicators of adiposity; however, most of the evidence is from observational studies carried out in adult populations ${ }^{(11-20)}$. It has been hypothesized that whole grains may potentially influence weight through possible effects on hormonal factors, satiety, and/or digestion and absorption of carbohydrates ${ }^{(21-24)}$. The Dietary Guidelines for Americans 2005 and 2010 include whole grains as one of the food groups to encourage in all age groups and recommends that children consume at least half of their daily servings of grain products as whole grains ${ }^{(25,26)}$. However, most cereal grain products consumed in the USA are refined and hence lower in fibre, vitamins, minerals and phytochemicals, among other nutrients, compared with wholegrain foods ${ }^{(27)}$. 
Evidence on the association between whole grain intake and body weight is limited in paediatric populations, even though grain foods are widely consumed by children $^{(28)}$. The objective of the present study was to examine the association between whole grain intake and BMI $Z$-score and prevalence and risk of overweight and obesity in rural children attending 3rd-6th grade who participated in the CHANGE study.

\section{Experimental methods}

\section{Study design and participants}

In 2005, Save the Children created the CHANGE (Creating Healthy, Active and Nurturing Growing-up Environments) Program with the objective of reducing unhealthy weight gain in school-aged children in rural America. To evaluate their efforts, a partnership between Save the Children and the Friedman School of Nutrition Science and Policy at Tufts University was formed and a randomized, controlled, community-based research study was conducted. The CHANGE I study recruited twenty-two randomly selected after-school programmes and the CHANGE II study recruited eight randomly selected elementary schools from rural communities in California, Kentucky, Mississippi and South Carolina. The CHANGE study was designed to (i) adapt, replicate and evaluate the Shape Up Somerville model, a multi-faceted environmental change intervention that used a community-based participatory research approach to address unhealthy weight gain among school-aged children living in an urban setting, and (ii) compare the CHANGE II multi-faceted intervention with the CHANGE I after-school intervention model ${ }^{(29)}$.

For the present cross-sectional study, anthropometric and dietary data collected at baseline in 3rd-6th grade children from all CHANGE II schools (spring 2008 and early autumn 2008) and CHANGE I after-school programmes (winter 2008) were used. Recruitment of participants and study procedures were approved and monitored by the Institutional Review Board at Tufts University. A total of 1501 children were enrolled after obtaining parental informed consent. Children were excluded from the analysis if: (i) the date on their consent form was later than the last day of data collection in the school ( $n$ 292); (ii) they were absent on the days when measurements were performed ( $n$ 128); and (iii) the date was omitted on either the parental consent ( $n$ 21) or data collection form ( $n$ 1). From the remaining 1059 children, 103 had missing BMI Z-score data and/or missing dietary data, leaving a total of 956 children. From these, fifteen had missing data on one or more of the covariates used for analyses (age, gender, race/ethnicity or physical activity) leaving 941 children. Although the Block Food Screener was not intended to assess energy intake, an estimation of energy intake was calculated for each participant with the purpose of identifying overreporters. There are no standard exclusion criteria for over- or under-reporting of food intake estimated by food screeners. For the purpose of the present study, implausible over-reporting of food intake was defined as estimated total energy intake $>20920 \mathrm{~kJ} / \mathrm{d}(5000 \mathrm{kcal} / \mathrm{d})$ and under-reporting of food intake as two or fewer food items being reported within a day. After exclusion of over-reporters ( $n$ 125) and under-reporters ( $n$ 24) the final sample size was reduced to 792 children.

\section{Outcome measures}

Anthropometry

Height and weight were obtained in triplicate following standardized procedures for school settings as previously described $^{(30,31)}$. Briefly, height was measured to the nearest $\sim 3 \mathrm{~mm}$ (1/8 in.) using a portable stadiometer (Shorr Infant/ Child/Adult Height/Length Measuring Board; Shorr, Olney, MD, USA). Weight was measured in light clothing to the nearest $\sim 225 \mathrm{~g}(0.5 \mathrm{lb})$ on a digital scale (Seca Bella model 480; Seca, Hanover, MD, USA). BMI Z-score was calculated using an average of three body weight measures divided by the average of three height measures and converted to $Z$-scores as recommended by the US Centers for Disease Control and Prevention ${ }^{(32)}$. Following these guidelines, a BMI Z-score $<5$ th percentile was considered underweight, a BMI $Z$-score $\geq 5$ th and $<85$ th percentile was considered normal weight, a BMI $Z$-score $\geq 85$ th percentile and $<95$ th percentile was considered overweight and a BMI $Z$-score $\geq 95$ th percentile was considered obese ${ }^{(32,33)}$.

\section{Diet assessment}

Diet was assessed using the 2007 Block Food Screener for ages $2-17$ years $^{(34)}$. This screener is designed to be selfadministered with adult assistance and captures intake and portion size for forty-one food and beverage items consumed over the previous $24 \mathrm{~h}$. It was developed and adapted from the validated Block Kids 2004 FFQ, an eighty-item questionnaire designed to assess food and nutrient intakes in children 2-17 years old ${ }^{(35)}$. The screener estimates daily servings of the following food groups: whole grains, fruits, vegetables (excluding potatoes), potatoes, meat/poultry/fish, dairy and legumes. The food list for this screener was created by identifying the most important sources of each of the food groups mentioned above in children aged 2-17 years as determined by data from two cycles of the National Health and Nutrition Examination Survey (NHANES 2001-2002 and 2003-2004). Food groups and portion sizes were selected for six age- and sex-specific categories. The Block Food Screener for ages 2-17 years has been used in various studies to assess food group intake in younger children ${ }^{(36)}$ and in children of similar age as the ones included in the present study ${ }^{(37,38)}$.

In the present study, the screener was completed by the children in small groups of five to ten with the assistance of a trained data collector. For each food and 
beverage included in the screener, the amount of each food group was determined by multiplying the reported frequency of consumption ( 1 if eaten yesterday; 0 if not eaten) by the age- and gender-specific portion size consumed (small, medium or large) and by the amount of each food group content in that food item. The total consumption of each food group was calculated by summing the amounts of each food group in each food item included in the screener. The primary resource for whole grain and other food group servings was the US Department of Agriculture MyPyramid Equivalents Database $^{(39)}$, which provides the number of MyPyramid equivalents (standard serving sizes) for the thirty-two MyPyramid major food groups and subgroups that are present in $100 \mathrm{~g}$ of each of the foods consumed by participants in NHANES. The MyPyramid whole grain group equivalents are defined in ounces. An ounce-equivalent or serving of whole grains is either $1 \mathrm{oz}(28 \mathrm{~g})$ of a $100 \%$ wholegrain food in its ready-to-eat form or the amount of food containing $16 \mathrm{~g}$ of wholegrain ingredients. On the screener, examples of a serving of whole grains include 1 cup of $100 \%$ wholegrain ready-to-eat cereal, half a cup of cooked oatmeal, a slice of $100 \%$ wholegrain bread, 3 cups of popcorn, etc. ${ }^{(26)}$. The associated screener database includes twelve sources of whole grains, which range in concentration from $0 \cdot 01$ to $3 \cdot 01$ servings of whole grains per $100 \mathrm{~g}$ of food item.

\section{Covariate measures}

Information on age, sex, race/ethnicity, grade and demographics was collected by a family survey, adapted from the Shape Up Somerville study, that was completed by the parents/primary caregivers ${ }^{(29)}$. Children completed a self-administered survey that included fourteen physical activity categories in the form of dichotomous variables of whether or not the child had performed each activity in the last week $^{(40)}$.

\section{Statistical metbods}

Statistical analyses were conducted using the SAS statistical software package version 9.2 (SAS Institute Inc.). Children were classified into three whole grain categories according to their daily intake: $<1 \cdot 0$ serving/d, $1 \cdot 0-1 \cdot 5$ servings/d and $>1.5$ servings/d. The age- and sexadjusted means for lifestyle and dietary characteristics were calculated across categories of whole grain intake using the SAS procedure PROC GLM. We assessed test for trend across categories of whole grain intake by assigning the median whole grain intake of each category to individuals in that category and treating this new variable as a continuous variable in the linear or logistic regression model. Linear regression was used for continuous and logistic regression for dichotomous lifestyle and dietary variables. To study the cross-sectional association between whole grain intake and body weight, we compared BMI $Z$-score means according to categories of whole grain intake using SAS PROC GLM program. Trends across whole grain intake categories were calculated as described above. We also calculated the adjusted prevalence of children classified as obese or overweight/obese within whole grain categories using linear regression. To further explore the association between whole grain intake and overweight/obesity, we calculated the odds ratios and associated $95 \%$ confidence intervals for obese and overweight/obese for each category of whole grain intake using multiple logistic regression. Children who consumed $<1 \cdot 0$ serving/d were used as the reference category. All models were adjusted for age and number of physical activities performed in the last week as continuous variables and sex, race/ethnicity and state of residence as categorical variables. Further adjustments for potential dietary predictors of body weight (fruit, vegetable and dairy intakes) were performed to assess the possibility that dietary patterns associated with diets rich in whole grains would explain the observed associations. We used Tukey adjustment to account for multiple comparisons between means. We created interaction terms to test whether the association between whole grain intake and BMI Z-score was modified by gender or age. No significant interaction was observed; therefore data were analysed combining all children.

\section{Results}

Participants were from families with a high household poverty level and were mostly non-white (>60\%). Over $20 \%$ of the children's primary household language was not English and an estimated one-third of parents had not graduated from high school. Out of 792 children with available data for analysis, sixty-nine (8.7\%) did not consume whole grains and only fifteen (1.9\%) consumed at least half of the recommended servings of grains as whole grains. The mean and median daily intakes of whole grains in this population of rural children were 0.8 and 0.6 servings/d respectively, with a median range that varied from 0.4 servings/d in the lowest whole grain intake category to $2 \cdot 0$ servings/d in the highest category. These estimated intakes are consistent with national data average intakes of 0.8 servings of whole grains/d in pre-school children and $1 \cdot 0$ servings/d in adolescents ${ }^{(41)}$. The main sources of whole grain intake in our study population were breakfast cereals (38\%), popcorn (28\%), wholegrain bread (18\%) and breakfast or protein bars $(15 \%)$, accounting for $99 \%$ of the total intake of whole grains.

Characteristics of the population by whole grain intake category are summarized in Table 1 . Children who consumed $>1.5$ servings of whole grains/d tended to be slightly younger, with a higher relative proportion of boys compared with girls, a higher proportion of Hispanic children, a higher proportion of children living in California 
Table 1 Characteristics of participants according to whole grain intake group: children ( $n$ 792) attending 3rd-6th grade, eight rural US communities in California, Mississippi, Kentucky and South Carolina, 2008 (CHANGE study)

\begin{tabular}{|c|c|c|c|c|}
\hline \multirow[b]{2}{*}{ Characteristic } & \multicolumn{3}{|c|}{ Whole grain intake (servings/d) } & \multirow[b]{2}{*}{$P$ trend $t$} \\
\hline & $<1 \cdot 0$ & $1 \cdot 0-1 \cdot 5$ & $>1.5$ & \\
\hline$n$ & 537 & 120 & 135 & - \\
\hline Median intake of whole grains (servings/d) & 0.3 & $1 \cdot 2$ & $2 \cdot 0$ & - \\
\hline Age (years) $\ddagger$ & $10 \cdot 4$ & $10 \cdot 2$ & $10 \cdot 2$ & 0.03 \\
\hline Female $(\%) \S$ & $61 \cdot 3$ & $53 \cdot 2$ & $51 \cdot 0$ & 0.01 \\
\hline \multicolumn{5}{|l|}{ Race/ethnicity (\%)\| } \\
\hline White & $23 \cdot 7$ & $14 \cdot 5$ & $18 \cdot 1$ & 0.05 \\
\hline Black & $40 \cdot 0$ & $34 \cdot 7$ & $36 \cdot 1$ & $0 \cdot 3$ \\
\hline Hispanic & $33 \cdot 7$ & $48 \cdot 3$ & $42 \cdot 9$ & 0.01 \\
\hline Other & $2 \cdot 6$ & $2 \cdot 5$ & $2 \cdot 9$ & $0 \cdot 9$ \\
\hline \multicolumn{5}{|l|}{ State $(\%) \|$} \\
\hline California & $37 \cdot 3$ & $51 \cdot 6$ & $47 \cdot 2$ & 0.01 \\
\hline Kentucky & $19 \cdot 9$ & $11 \cdot 9$ & $15 \cdot 9$ & $0 \cdot 1$ \\
\hline Mississippi & $19 \cdot 7$ & $21 \cdot 0$ & $20 \cdot 3$ & $0 \cdot 8$ \\
\hline South Carolina & $23 \cdot 0$ & $15 \cdot 5$ & $16 \cdot 6$ & 0.04 \\
\hline Number of physical activities $\|$ & $3 \cdot 7$ & $3 \cdot 8$ & $4 \cdot 4$ & 0.04 \\
\hline Fruit intake (servings/d)\| & $1 \cdot 8$ & $2 \cdot 5$ & $2 \cdot 7$ & $<0.001$ \\
\hline Vegetable intake (servings/d)\| & $1 \cdot 3$ & $2 \cdot 0$ & $2 \cdot 3$ & $<0.001$ \\
\hline Dairy intake (servings/d)\| & $1 \cdot 8$ & $2 \cdot 6$ & $2 \cdot 8$ & $<0.001$ \\
\hline
\end{tabular}

CHANGE, Creating Healthy, Active and Nurturing Growing-up Environments.

tFor continuous variables, the median value in each whole grain intake category was used as a continuous variable in a linear regression; for categorical variables, logistic regression was used.

†Mean, adjusted for sex.

\$Mean, adjusted for age.

\|Mean, adjusted for age and sex.

-Number of physical activities performed in the last week from a self-administered survey that included fourteen physical activity categories.

Table 2 Multivariate-adjusted geometric mean BMI Z-score and prevalence, odds ratio and $95 \%$ confidence interval of overweight and obesity according to whole grain intake group: children $(n 792)$ attending 3rd-6th grade, eight rural US communities in California, Mississippi, Kentucky and South Carolina, 2008 (CHANGE study)

\begin{tabular}{lcccr}
\hline & \multicolumn{3}{c}{ Whole grain intake (servings/d) } & \\
\cline { 2 - 4 } & $<1.0$ & $1.0-1.5$ & $>1.5$ & $P$ trendt \\
\hline$n$ & 537 & 120 & 135 & - \\
Median intake of whole grains (servings/d) & 0.3 & 1.2 & 2.0 & 0.01 \\
BMI Z-scoreł & 0.90 & 0.89 & $0.61^{*}$ & 0.06 \\
Obese & 31.3 & 33.0 & 21.4 & 0.07 \\
$\quad$ Prevalence (\%) & 1.00 & 1.10 & $0.60^{\star *}$ & 0.09 \\
OR & Ref. & $0.71,1.67$ & $0.38,0.95$ & 0.08 \\
95\% Cl & 46.0 & 45.0 & 36.8 & 0.69 \\
Overweight/obese & 1.00 & 0.96 & $0.46,1.01$ & \\
Prevalence (\%) & Ref. & $0.64,1.44$ & & \\
OR & & &
\end{tabular}

CHANGE, Creating Healthy, Active and Nurturing Growing-up Environments; Ref., referent category.

All analyses were adjusted by age, sex, race/ethnicity, state of residence and physical activity. Significant differences remained statistically significant after further adjustments for fruit, vegetable and dairy intakes.

${ }^{\star} P=0.01$ compared with the group consuming $<1.0$ serving of whole grains $/ \mathrm{d}$; ${ }^{\star *} P=0.02$ compared with the group consuming $<1.0$ serving of whole grains/d.

tFor continuous variables, the median value in each whole grain intake category was used as a continuous variable in a linear regression; for categorical variables, logistic regression was used.

$\ddagger$ Adjusted mean.

and a lower proportion of children living in South Carolina. Greater intake of whole grains was also associated with significantly higher number of physical activities/week and intakes of fruits, vegetables and dairy products.

The relationship between whole grain intake and BMI $Z$-score is shown in Table 2. After adjustment for age, sex, state of residence, race/ethnicity and physical activity, a significant inverse association was observed between whole grain intake and BMI Z-score $(0.90 v .0 .61$ in the lowest $v$. the highest whole grain intake category; $P$ trend $=0 \cdot 01$ ). The difference between the mean BMI $Z$-score in the lowest and the highest whole grain intake category $(0 \cdot 29)$ can be best understood if we consider an example of a 10-year-old boy with an intake of whole grains in the highest category and with height and weight at the 50 th percentile $(138 \cdot 8 \mathrm{~cm}$ and $32 \cdot 09 \mathrm{~kg}$, respectively). 
For this reference child, a difference of $0 \cdot 29$ in BMI $Z$-score would correspond to an approximate difference of $1.2 \mathrm{~kg}$ $(2 \cdot 7 \mathrm{lb})$; in other words, a boy with the same height but with a whole grain intake in the lowest category would theoretically have a weight that is $1 \cdot 2 \mathrm{~kg}(2 \cdot 7 \mathrm{lb})$ higher. Further adjustment for potential dietary predictors of body weight (fruit, vegetable and dairy intakes) did not substantially change the association between whole grain intake and BMI $Z$-score $(P$ trend $=0 \cdot 03)$.

In this study population, $48 \%$ of children were categorized as overweight or obese and 30\% were categorized as obese. Table 2 displays the prevalence and risk of obesity and overweight/obesity by whole grain intake category adjusted by age, sex, race/ethnicity, state of residence and number of physical activities performed in the last week. Across categories of whole grain intake, an inverse association was observed between whole grain intake and the percentage of children categorized as obese or overweight/obese, although these associations did not reach statistical significance $(P$ trend $=0.06$ and 0.09 , respectively). However, the odds of being obese were lower with higher intake of whole grains. Compared with children who consumed $<1 \cdot 0$ serving of whole grains/day, those who consumed $>1.5$ servings/d had a $40 \%$ lower risk of being obese (OR $=0 \cdot 60 ; 95 \%$ CI $0 \cdot 38,0 \cdot 95, P=0 \cdot 02)$. Further adjustments for fruit, vegetable and dairy intakes did not substantially change this association $(P=0 \cdot 02)$.

\section{Discussion}

With the staggering prevalence of overweight and obesity in children, there is an increased need to identify lifestyle factors that could potentially promote the attainment and maintenance of a healthy weight. Given the particularly high rates of obesity among rural youth and the need to explore modifiable dietary factors, we sought to assess the association between intake of whole grains and BMI $Z$-score, prevalence and risk of overweight and obesity in rural children participating in the CHANGE study. In this group of children, we identified significant inverse associations between whole grain intake and BMI $Z$-score and risk of obesity. Children who consumed $>1.5$ servings of whole grains/d (approximately half of the recommended amount) had a significantly lower BMI $Z$-score and were less likely to be obese even after adjustments for other lifestyle and dietary covariates compared with children who consumed $<1 \cdot 0$ serving/d.

Our finding that whole grain intake is inversely associated with body weight is consistent with the findings reported in the literature, albeit primarily in adults ${ }^{(11-14,16-20,42,43)}$. Fewer studies have examined the impact of whole grains on body weight in children or adolescents and the results are conflicting. In adolescents, Cheng et al. reported in 215 children (mean age $9 \cdot 4$ years) no association between whole grain intake and percentage body fat or BMI Z-score at baseline or between changes in whole grain intake and changes in percentage body fat or BMI $Z$-score over 4 years ${ }^{(4)}$. Davis et al. reported that changes in fibre intake, but not whole grain intake, were associated with changes in visceral adiposity in a small sample of eighty-five overweight Latino adolescents aged $11-17$ years $^{(45)}$. Steffen et al., on the other hand, reported that whole grain intake was associated with lower BMI in 285 adolescents $^{(15)}$. Using data from the NHANES 1999 and 2004, Zanovec et al. found a direct association between consuming 1.5 to 3 servings of whole grains daily and weight measures in 3868 children aged 6-12 years, but an inverse association between consuming at least 3 servings of whole grains daily and BMI Z-scores in 4931 adolescents aged 13-18 years ${ }^{(46)}$. The inconsistency of these findings may be due to differences in study design, dietary assessment methods, prevalence of overweight/ obesity in the populations studied or lack of power to detect associations, among others.

There are several possible mechanisms by which intake of whole grains may be protective against obesity. The high fibre content and overall lower glycaemic index of food containing whole grains may increase satiety and result in decreased energy consumption ${ }^{(22-24,47-49)}$. In addition, wholegrain foods are generally less energydense than refined-grain foods due to the high fibre and water content ${ }^{(21,50)}$. Furthermore, the intake of wholegrain food may result in a delayed digestion and absorption of its starch and thus result in relatively lower postprandial insulin and glucose responses ${ }^{(22-24,51)}$. As high insulin levels may promote storage of metabolic substrates, a lower insulin response may result in the oxidation and lipolysis of fat rather than its storage ${ }^{(24)}$.

One recognized limitation of this type of analysis is that often people with diets rich in whole grains tend to follow healthier diets and lifestyles, making it difficult to attribute health outcomes directly to whole grain intake. To address this issue, we adjusted our analyses for potential confounding dietary and lifestyle factors including the number of physical activities children reported participating in over the past week and intakes of fruits, vegetables and dairy products. After adjustments, observed associations between whole grain intake and BMI Z-score and obesity remained. However, the possibility of residual confounding cannot be excluded in any observational study. In addition, the cross-sectional nature of the study precludes any inferences on the direction of our associations, making it impossible to determine whether changes in whole grain intake precede changes in body weight. A recognized limitation is that the assessment of diet in children is particularly challenging. The Block Food Screener used in the present study was specifically designed to calculate number of servings of whole grain intake in this age group ${ }^{(34)}$. This screener assessed a limited number of foods consumed on a single day and was not designed to calculate energy intake, which hindered our ability to adjust for total energy intake. However, total energy may potentially be one of the 
mechanisms by which intake of whole grains is associated with weight, in which case adjustment for total energy would not be appropriate. Lastly, because very few children consumed at least half of their recommended servings of grains as whole grains ${ }^{(25,26)}$, we were not able to study the effect of meeting this recommendation on measures of body weight.

\section{Conclusions}

In the current cross-sectional analysis of children living in rural communities with greater than average prevalence of overweight/obesity, intake of whole grains was inversely associated with BMI Z-score and obesity. Despite current recommendations, consumption of whole grains is very low in this population. These results suggest that increasing the intake of foods rich in whole grains as part of an overall healthy lifestyle may be beneficial for children to maintain a healthy weight. Efforts should be made to increase the availability of foods rich in whole grains especially to lowincome populations and to improve the general public's knowledge to identify foods containing whole grains. Longitudinal and intervention studies are needed to further evaluate the effect of whole grain consumption on body weight in children.

\section{Acknowledgements}

Source of funding: This work was supported by the following grants from the Save the Children Foundation: 'A Partnership for CHANGE' and 'The CHANGE II Study: Obesity Prevention in Rural America'. Conflicts of interest: S.F.C., A.T., R.R.H., A.V.H., J.B.H. and C.D.E., none. At the time of the study, V.I.K. was employed by Save the Children USA, which had received funding from America Gives Back, the PepsiCo Foundation, Kraft Foods, Cadbury North America and the General Mills Foundation; she has no conflicts in her current position as a PhD candidate at Deakin University in Melbourne, Victoria, Australia. N.M.M. is supported in part by a research grant from General Mills Bell Institute of Health and Nutrition, Minneapolis, MN, USA. Author contributions: S.F.C., N.M.M. and C.D.E. designed the present study; A.V.H., J.B.H. and C.D.E. were responsible for collection of the data; S.F.C., N.M.M. and C.D.E. conducted the analysis; and S.F.C., N.M.M., A.T., R.R.H., V.I.K., A.V.H., J.B.H. and C.D.E. interpreted the analysis, contributed to writing the manuscript and made substantial conceptual contributions and revisions.

\section{References}

1. Ogden CL, Yanovski SZ, Carroll MD et al. (2007) The epidemiology of obesity. Gastroenterology 132, 2087-2102.

2. Ogden CL, Carroll MD, Curtin LR et al. (2010) Prevalence of high body mass index in US children and adolescents, 2007-2008. JAMA 303, 242-249.
3. South Carolina Rural Health Research Center (2007) Overweight and Physical Inactivity among Rural Children Aged 10-17: A National and State Portrait. http://rhr.sph.sc.edu/ report/(7-1)Obesity\%20ChartbookUpdated10.15.07-secured. pdf (accessed September 2011).

4. Tudor-Locke C, Kronenfeld JJ, Kim SS et al. (2007) A geographical comparison of prevalence of overweight school-aged children: the National Survey of Children's Health 2003. Pediatrics 120, e1043-e1050.

5. Lutfiyya MN, Lipsky MS, Wisdom-Behounek J et al. (2007) Is rural residency a risk factor for overweight and obesity for US children? Obesity (Silver Spring) 15, 2348-2356.

6. Serdula MK, Ivery D, Coates RJ et al. (1993) Do obese children become obese adults? A review of the literature. Prev Med 22, 167-177.

7. National Institutes of Health (1998) Clinical guidelines on the identification, evaluation, and treatment of overweight and obesity in adults - The Evidence Report. Obes Res $\mathbf{6}$, Suppl. 2, 51S-209S.

8. US Surgeon General (2001) Overweight and Obesity: health consequences. http://www.surgeongeneral.gov/ library/calls/obesity/fact_consequences.html (accessed July 2012).

9. Renehan AG, Tyson M, Egger M et al. (2008) Body-mass index and incidence of cancer: a systematic review and meta-analysis of prospective observational studies. Lancet 371, 569-578.

10. Olshansky SJ, Passaro DJ, Hershow RC et al. (2005) A potential decline in life expectancy in the United States in the 21st century. $N$ Engl J Med 352, 1138-1145.

11. Sahyoun NR, Jacques PF, Zhang XI et al. (2006) Whole-grain intake is inversely associated with the metabolic syndrome and mortality in older adults. Am J Clin Nutr 83, 124-131.

12. Liu S, Willett WC, Manson JE et al. (2003) Relation between changes in intakes of dietary fiber and grain products and changes in weight and development of obesity among middle-aged women. Am J Clin Nutr 78, 920-927.

13. Koh-Banerjee P, Franz M, Sampson L et al. (2004) Changes in whole-grain, bran, and cereal fiber consumption in relation to 8-y weight gain among men. Am J Clin Nutr 80, $1237-1245$.

14. McKeown NM, Meigs JB, Liu S et al. (2002) Whole-grain intake is favorably associated with metabolic risk factors for type 2 diabetes and cardiovascular disease in the Framingham Offspring Study. Am J Clin Nutr 76, 390-398.

15. Steffen LM, Jacobs DR Jr, Murtaugh MA et al. (2003) Whole grain intake is associated with lower body mass and greater insulin sensitivity among adolescents. Am J Epidemiol 158, 243-250.

16. Newby PK, Maras J, Bakun P et al. (2007) Intake of whole grains, refined grains, and cereal fiber measured with 7-d diet records and associations with risk factors for chronic disease. Am J Clin Nutr 86, 1745-1753.

17. Lutsey PL, Jacobs DR Jr, Kori S et al. (2007) Whole grain intake and its cross-sectional association with obesity, insulin resistance, inflammation, diabetes and subclinical CVD: The MESA Study. Br J Nutr 98, 397-405.

18. Good CK, Holschuh N, Albertson AM et al. (2008) Whole grain consumption and body mass index in adult women: an analysis of NHANES 1999-2000 and the USDA pyramid servings database. J Am Coll Nutr 27, 80-87.

19. McKeown NM, Yoshida M, Shea MK et al. (2009) Wholegrain intake and cereal fiber are associated with lower abdominal adiposity in older adults. J Nutr 139, 1950-1955.

20. van de Vijver LP, van den Bosch LM, van den Brandt PA et al. (2009) Whole-grain consumption, dietary fibre intake and body mass index in the Netherlands cohort study. EurJ Clin Nutr 63, 31-38.

21. Koh-Banerjee P \& Rimm EB (2003) Whole grain consumption and weight gain: a review of the epidemiological 
evidence, potential mechanisms and opportunities for future research. Proc Nutr Soc 62, 25-29.

22. Jenkins DJ, Jenkins AL, Wolever TM et al. (1987) Starchy foods and fiber: reduced rate of digestion and improved carbohydrate metabolism. Scand J Gastroenterol Suppl 129, 132-141.

23. Jenkins DJ, Wesson V, Wolever TM et al. (1988) Wholemeal versus wholegrain breads: proportion of whole or cracked grain and the glycaemic response. BMJ 297, 958-960.

24. Slavin JL, Martini MC, Jacobs DR Jr et al. (1999) Plausible mechanisms for the protectiveness of whole grains. $A m J$ Clin Nutr 70, 3 Suppl., 459S-463S.

25. US Departmant of Health and Human Services \& US Department of Agriculture (2005) Dietary Guidelines for Americans, 6th ed. Washington, DC: US Government Printing Office.

26. US Departmant of Health and Human Services \& US Department of Agriculture (2010) Dietary Guidelines for Americans, 7th ed. Washington, DC: US Government Printing Office.

27. Putnam J, Allshouse J \& Kantor L (2002) US per capita food supply trends: more calories, refined carbohydrates, and fats. Food Rev 25, 2-15.

28. Newby PK (2009) Plant foods and plant-based diets: protective against childhood obesity? Am J Clin Nutr 89, issue 5, 1572S-1587S.

29. Economos CD, Hyatt RR, Goldberg JP et al. (2007) A community intervention reduces BMI Z-score in children: Shape Up Somerville first year results. Obesity (Silver Spring) 15, 1325-1336.

30. Michigan Department of Education (2001) The Role of Michigan Schools in Promoting Healthy Weight: A Consensus Paper. East Lansing, MI: Michigan Department of Education.

31. Lohman T (1992) Advances in Body Composition Assessment. Champaign, IL: Human Kinetics Publishers.

32. Centers for Disease Control and Prevention (2000) CDC table for calculated body mass index values for selected heights and weights for ages 2 to 20. http://www.cdc.gov/ growthcharts/html_charts/bmiagerev.htm (accessed July 2012).

33. Barlow SE (2007) Expert committee recommendations regarding the prevention, assessment, and treatment of child and adolescent overweight and obesity: summary report. Pediatrics 120, Suppl. 4, S164-S192.

34. NutritionQuest (2007) Block Food Screeners for Ages 2-17. http://www.nutritionquest.com/assessment/list-ofquestionnaires-and-screeners (accessed September 2011).

35. Cullen KW, Watson K \& Zakeri I (2008) Relative reliability and validity of the Block Kids Questionnaire among youth aged 10 to 17 years. J Am Diet Assoc 108, 862-866.

36. Murashima M, Hoerr SL, Hughes SO et al. (2011) Confirmatory factor analysis of a questionnaire measuring control in parental feeding practices in mothers of Head Start children. Appetite 56, 594-601.

37. Davis JN, Ventura EE, Cook LT et al. (2011) LA Sprouts: a gardening, nutrition, and cooking intervention for Latino youth improves diet and reduces obesity. J Am Diet Assoc 111, 1224-1230.

38. Garcia-Dominic O, Trevino RP, Echon RM et al. (2011) Improving quality of food frequency questionnaire response in low-income Mexican American children. Health Promot Pract (Epublication ahead of print version).

39. Friday JE \& Bowman SA (2006) MyPyramid Equivalents Database for USDA Survey Food Codes, 1994-2002 Version 1.O. Beltsville, MD: USDA, ARS, Community Nutrition Research Group; available at http://www.ars.usda.gov/ $\mathrm{ba} / \mathrm{bhnrc} / \mathrm{fsrg}$

40. National Recreation and Park Association \& National Heart, Lung, and Blood Institute (2004) Hearts N' Parks - Report of 2004 Magnet Center Performance Data. http://www. nhlbi.nih.gov/health/prof/heart/obesity/hrt_n_pk/2004_ report.pdf (accessed September 2011).

41. Harnack L, Walters SA \& Jacobs DR Jr (2003) Dietary intake and food sources of whole grains among US children and adolescents: data from the 1994-1996 Continuing Survey of Food Intakes by Individuals. J Am Diet Assoc 103, 1015-1019.

42. Katcher HI, Legro RS, Kunselman AR et al. (2008) The effects of a whole grain-enriched hypocaloric diet on cardiovascular disease risk factors in men and women with metabolic syndrome. Am J Clin Nutr 87, 79-90.

43. Jonnalagadda SS, Harnack L, Liu RH et al. (2011) Putting the whole grain puzzle together: health benefits associated with whole grains - summary of American Society for Nutrition 2010 Satellite Symposium. J Nutr 141, issue 5, 1011S-1022S.

44. Cheng G, Karaolis-Danckert N, Libuda L et al. (2009) Relation of dietary glycemic index, glycemic load, and fiber and whole-grain intakes during puberty to the concurrent development of percent body fat and body mass index. Am J Epidemiol 169, 667-677.

45. Davis JN, Alexander KE, Ventura EE et al. (2009) Inverse relation between dietary fiber intake and visceral adiposity in overweight Latino youth. Am J Clin Nutr 90, 1160-1166.

46. Zanovec M, O'Neil CE, Cho SS et al. (2010) Relationship between whole grain and fiber consumption and body weight measures among 6- to 18-year-olds. J Pediatr 157, 578-583.

47. Howarth NC, Saltzman E \& Roberts SB (2001) Dietary fiber and weight regulation. Nutr Rev 59, 129-139.

48. Roberts SB \& Heyman MB (2000) Dietary composition and obesity: do we need to look beyond dietary fat? J Nutr 130, 2S Suppl., 267S.

49. Marlett JA, McBurney MI \& Slavin JL (2002) Position of the American Dietetic Association: health implications of dietary fiber. J Am Diet Assoc 102, 993-1000.

50. Pereira MA, Jacobs DR Jr, Pins JJ et al. (2002) Effect of whole grains on insulin sensitivity in overweight hyperinsulinemic adults. Am J Clin Nutr 75, 848-855.

51. Lundin EA, Zhang JX, Lairon D et al. (2004) Effects of meal frequency and high-fibre rye-bread diet on glucose and lipid metabolism and ileal excretion of energy and sterols in ileostomy subjects. Eur J Clin Nutr 58, 1410-1419. 